\title{
Communication, Conflict and Crisis Management
}

\author{
Tina C. Touitou
}

\begin{abstract}
Communication is an indispensable tool in business and in all human organization. This is to say that no organization can exist or succeed without effective communication. Also, of great relevance when it has to do with managing conflicts and cries. In human relation there is bound to be conflicts due to differences in the way people perceive things, their divergent interest needs, goals and personalities. No society can survive without conflict but can thrive better under discordant voices. Conflict is inevitable in human society, whether formal and informal conflicts or crises, can be managed and resolved through effective communication. Based on these the paper adopted three conflict models: Structural /Six Hubs Wheel Model of Conflict Management, the Structural and Process Model, Skchammer and Conflict Management Model, it also employed empirical secondary data. The finding of the study opines that effective communication has been proven by research and experience to be at the heart or centre of effective crisis management. It further concluded that the best antidote in crisis management techniques is consistent and persistent communication, with consistent communication and thoughtful planning, one is better prepared to counter crisis successfully.
\end{abstract}

Index Terms - Communication, Conflict, Crisis, Crisis Management.

\section{INTRODUCTION}

Communication involves the use of signs, signals, symbols and language. As human beings, we communicate by any of these means. At a much larger spectrum, we employ machines or mechanical devices to communicate with a diverse and mass of people through the use of television, radio, newspaper, magazines and films. Communication occurs in a variety of contexts for example, interpersonal, small group, organizational, community, and mass media) with a variety of messages and for a variety of reasons.

Lester [1] asserts that one of the first forms of communication among Homo sapiens was by speech. This helped in the transmission of information and knowledge from one generation to another. Speech is however not perfect, as it relies on the memory of individuals- an imperfect tool in the dissemination of knowledge, as it can change, be corrupted because there is the tendency to forget and a limit to how much one can remember. Interpersonal relationship has its roots in communication. Communication has its root from two words, "com" (from the Latin word) "cum" - "which means to establish a community or commonness or to share"

$\mathrm{He}$ further, observed that communication serves as an instrument of social interaction; it helps us to understand ourselves; to keep in touch with other people; to understand situations. It is a means by which power is acquired; exercised and sustained, and the medium through which relationships are established, extended, and maintained. Communication is the bedrock of all that we do and is the vital element of human existence. Without communication, man cannot have knowledge. Communication, which is the process of transferring thoughts, ideas, facts, feelings, etc from one person to another is very important in human existence, while, without it, life will be meaningless and human interaction would be impossible.

Anatsui [2] cited Black 1989 asserts that the major cause of conflict is misunderstanding. So, to resolve conflict means to achieve an understanding. The achievement of understanding is largely dependent on information, education and communication. Touitou [3] contends that "wherever man exists, communication must take place and the communication must be planned. It must be ordered to be effective, and it helps cooperation and understanding". It is to be understood that the aim of any organization or country, as to ensure that all concerned (external and internal publics) have a better understanding of the institution's policies and the "modus operandi" of executing such policies.

Kunle [4] asserts that "the message from the source should be well articulated so that the receiver or audience who has expectations concerning an issue of importance can develop consent'. The audience affected by the set objective must be identified, analyzed, and classified according to their interest. This is because people cannot be carried along without their consent and the support of any given cause must emanate from understanding that marries the articulation of the message with expectations. Research finding recognized that a proactive approach is the best philosophy for addressing issues that lead to crisis. Supporting this view, Anatsui [2] said that "the best tool for managing crisis is making sure that crisis does not arise, by using the regular planned and sustained program of communication.

\section{CONCEPT ANALYSIS, DISCUSSION AND EXPLANATIONS}

\section{A. Concept of Communication}

Communication is defined as "the transfer of information from one place to another place in a desired direction with an expectation to bring about a desired effect. Communication is transfer of idea of knowledge in ways that enable the recipients to understand, react to and to act upon the information received." However, for the receiver of messages to react accordingly, communication must be effective. To achieve this end, a thorough research must always be conducted by communicators both the media used and its effects. The study of communication effects via providing a platform for interaction between senders and receivers; while 
that of the media would reveal the suitability of the media selected for the audiences.

Onekutu \& Ojebode [5] corroborated this, saying that "... communication research is not just studying how message gets from one point to another, it focuses on how people use message to inform, persuade, manage, relate and influence one another in various contexts and cultures using a variety of channels and media.

Moemeka [6] also gives a note of warning to some communicators who depend singly on mass media to achieve real impact on the publics, reiterating what research has said in this regard that: "Important though the media because of their potential for area coverage and their impact on attitude change and behavior, they are usually not able to succeed in changing people's attitude, let alone cause them to behave according to the demands of message. Therefore, mass media efforts must be supplemented with interpersonal communication in order to assure success.

Moemeka further opines that in view of lapse, proposes an integrated communication strategy that" ... combines interpersonal and mass media strategies, carefully eliminating their limitations and maximizing their potentials and strengths; the strategy, using the media, feeds the interpersonal and traditional networks within the audience relevant information that would generate positive discussions which would lead to intelligent understanding of how differences can be resolved similarities, and harmonized to live in a crisis free environment".

In view of the following, communication theory admits that for effective targeting of the audiences and achieving the desired impact, the message must be compatible with the vehicle' that conveys it to the audiences. According to Macluhan, the medium is message; that is, the medium certainly influences the effects of message on - target audiences. So, careful selection of medium is of utmost importance achieving the desired effects. This simply means that not all messages fit into the mass media. Communication theory also makes one believes that interpersonal communication is the best in most cases, most especially in conflicts resolution and crises management because of its obvious advantages and effects within such contexts.

It is only in the interpersonal communication context that Communicant could achieve instantaneous feedback which eventually reveal the effects of the process. However, communicators should be careful not to confine the concept of interpersonal communication to verbal communication which is only an integral part of its constituents. In reality, interpersonal communication incorporates other methods of communication at all level of communication. This is because in our everyday lives, we fail to realize that we utilize other modes of communication other than formal speech, most of which, ordinarily are not aware of, but which are of utmost importance to us.

Our attention tends to be focused upon words and .... sentences rather than upon gestures, mannerism, or the way in which we space ourselves. The implication of this to us as communicators is that we have much relied on speech which is communication through a learned, organized, system of patterned vocal response which has been so developed and so efficient to us in our social interactions or group and public communications that we have often thought it to operate to the exclusion of all other modes of communication. This neglect, by implication, has an adverse effect on our ability and competence as persuasive communicators

Besides, for effective communication in conflicts resolution and crises management, communicators should know what constitutes conflicts and crises in all its' ramifications. For instance, Crises and conflicts could be covert or overt in nature just like communication itself. By overt communication, we mean verbal communication that is highly intended and highly noticeable through the uses of speech and other forms. Covert communication, on the other hand, is the manifest, but least considered aspects of our communication like the unintended gestures; mannerisms; ways of speaking; spacing .... etc. These we fail to manage and supplement with our overt communication to produce targeted persuasive effects.

And it has negative effect on our communication just because we tend to only imperfectly aware of our covert communication and thus, it's difficult for us to bring under expressive control. This implies that a great deal of discrepant communication undoubtedly occurs because we tend to say one overtly (verbally) but say another thing covertly (behaviorally). And co-ordination of these two modes of communication, between communicants, is crucial for effective communication. The ability to coordinate these two modes of communication in crisis between communicants is thus crucial for effective communication. The inability to coordinate these two modes of communication in crisis communication would make it difficult for communicators, as reconciliators or negotiators, to manage crisis effectively and solve conflict situations. The negative consequence of failing to synergize verbal and non-verbal elements in conflict resolutions exemplified by children who when ask a question will say "yes" (overt communication) and then shake their heads to say "no" (covert communication) this, causing what is referred to as communication dissonance.

Besides, covert communication methods are communication through touch; proxemics, or communication through the handling of space; kinesics, or communication through motion of the body and limbs. Going by the foregoing, the question that arises is: how many of us as individuals and persuasive communicators manage and use these modes of communication in mundane conversations, let alone in crisis communication? To drive this point home, let us look at how covert communication was used effectively in a crisis situation.

Of course, we all remember the Oklahoma bombing during the former president Bill Clinton' administration. What happened then was a terrorist attack on school children which expectedly created a crisis situation. Immediately after the attack, the president was at the scene wittily applied covert communication principles - weeping profusely- kinesicsshowing sympathy, and concern to victims; closing the gap between himself and the publics-proxemics, as well a touching and caressing the victims-tactile. Is that not adding human touch which has its own distinct effects on effective communication? And is it not a perfect coordination of both overt and covert communication? 


\section{B. Principles of Effective Communication}

The basic principles in communication to be effective are follows:

- Completeness: A message is not complete if it is not receiver conscious. In order to make the message meaningful, communication must be complete. All necessary information that is needed to produce the entire effect of the message should be provided. For example, in pictorial communication, all the charts, graphs and diagrams must be properly and fully labeled.

- Conciseness: There must be brevity without jeopardizing completeness and courtesy. The words used must be exact and straight to the point. Explanation of terms must be short and precise.

- Clarity: The concept of the message should be clearly presented. Complicated use of words that can distort the message should be avoided. Grammatical expression must be carefully constructed in order to bring the intended meanings out.

- Courtesy: This means respect and politeness. This includes, prompt answer mails (if possible 23 days), avoiding expressions that hurt and belittle, apologizing for offences committed and the use of appreciating words.

- Concreteness: This means being specific, definite and vivid rather than vague $=$ general. The words used should not be ambiguous and abstract.

- Consideration: The needs and the demands of the audience must be considered be communication can be effective.

- Correctness: These tests all the six already mentioned principles. When all the : principles are complied with, communication is said to be correct when the message/information is complete, concise, clear, concrete, contains courtesy and considers the receiver, the message/information is said, be correct and the correctness also has to do with the accuracy of the message.

The Role of Communication in Conflict: Communication and conflict have a causal relationship. As a result of communication, the conflict might arise, but communication plays a causal preventive and curative role in conflict situations.

Communication as a Casual Factor in Conflict: This explains that conflict can occur during human interaction which is caused by communication between people in an organization. When there is an open discussion, there is the possibility that there will be differences. This shows that communication is a causal factor in conflict.

Communication as a Conflict Preventing Factor: Communication could also help to prevent conflict in an organization and to achieve this; one must foster supportive and participatory communication mood within the organization. If the organization climate is one of trust, confidence and openness, the conflict will rarely occur.

Communication as a Conflict Curative Factor: Communication also serves as a curative factor in conflict situations. This means it helps in managing conflict in an organization and plays an important role in resolving the conflict. This will lead us to define conflict resolution and conflict management

\section{Synergistic Communication Model}

The term 'synergy' is gotten from the Greek word 'synergia', which means 'work together'. In other words, it is the working together of two or more people, organizations or things especially when the result is greater than the sum of their individual effects or capabilities. The public relations synergistic communication strategies can be used in groups as their management principles. They are guided by the principle that the whole is greater than its various parts and units. It prompts its communication to be selfless, empathetic, less defensive, less judgmental and or legalistic and be more caring, friendly, altruistic and welcoming.

Nwosu \&Wilson [7], identified four strategies of synergistic communication as follows:

- Adopting the empathetic communication strategy.

- Adopting the Ethos + Pathos + Logos strategy.

- Adopting the Total Synergistic Communications strategy.

- Adopting the information and NCTS Mastery strategy.

Adopting the Empathetic Communication Strategy: The word 'empathetic' means putting yourself in someone else's shoes to feel their difficulties and interact on a more personal level. It means that the communicator should consider the target audience before himself; this can be very useful in tailoring your messages to suit their needs and ideologies. It seeks convergence and not a divergence in carrying out its task of uniting a flow of information in the organization.

Adopting the Ethos + Pathos + Logos Strategy: This strategy is a careful integration of ethos, pathos and logos principle. Ethos has to do with one's integrity and your credibility as an individual. Pathos deals with the feelings you send towards people and how they perceive those feelings, your ability to identify the problems of your public. And a Logo deals with the logical content of your message. This strategy begins with self-evaluation and analysis (Ethos). It simply means that you should identify with others (Pathos) and be logical and sensible in carrying all these out.

Adopting the Total Synergistic Communications Strategy: The total synergistic communication approach is a branch of Total Quality Management (TQM). It teaches that the entire organization should be carried along in the achievement of tasks and emphasizes on the adequate mobility of the work force of the organization. It is based on trust and less suspicion and also helps build team work and unity among all workers.

Adopting the Information and NCTS Mastery Strategy: NCTS refers to the New Communication Technologies. It constitutes the computer-based technologies that assist the managers to make good decisions concerning his department and ultimately the organization. It includes educating ourselves to use the NCTs and educating others below us to optimize everyone being on the same level (synergy).The adequate use of these tools will promote productivity, better inter-office relations and multiplication of workers capabilities in various ways. It would save time and resources for the organization. This would ultimately boost their interrelations to serve the society better through 
adopting synergistic communication in the crisis management plans.

\section{Concept of Conflict and Conflict Resolution}

Conflict. Darling and Walker [8] note that a 'conflict is a situation in which two or more individuals operating within a unit appear to be incompatible'. A resolution is an act of finding a satisfactory way of dealing with a problem or difficulty. Conflict resolution is but a part of conflict management which is wider in scope. Many conflict resolution techniques can be employed at different stages of managing conflict and at various levels which will be discussed later. Kolb and Putnam [9] situate conflict to arise due to perceptual or real dissimilarity in situations. The various definitions of conflict necessitated the grouping of conflict conceptualization into three schools of thought: Traditional, Human relations and Integrationist views.

\section{E. Three Conflict School of Thought}

Traditional Scholars View: see conflict to represent a negative phenomenon that must be avoided at all cost and also a sign of a problem. This line of reasoning favours adopting a simplistic approach to conflict management by merely directing attention to the causes of conflict and how to correct malfunctions to improve group and organizational performance [10].

Human Relations Perspective: regards conflict to be a natural occurrence in every organization and it should not necessarily be regarded as a negative phenomenon, and that conflict should be accepted. Thus, instead of the conflict avoidance that characterized the traditional view, this view opted for conflict management as a deliberate strategy to harness the positive outcome of conflict to enhance individual and organizational performance.

Integrationist View: goes a step further by not only accepting the imperative of conflict in organizations but to encourage conflict, because a harmonious, peaceful, tranquil, and cooperative group is prone to becoming static, apathetic and non-responsive to needs for change and innovation, Robbins [10] opines that the view of Integrationists did not intend to imply that all conflicts are desirable. In relations to this, the conflict has been discussed under two broad categories namely: functional and dysfunctional conflicts. Functional conflicts are those conflicts, which work to improve the performance of the organization. While dysfunctional conflicts destabilize the organization and could lead to sub-optimal organizational operation. The choice is to cultivate and nurture functional conflicts while if possible avoid structures and processes that could generate dysfunctional conflict. Hage [10], contends that organization conflict means "disagreement between the organizational means or ends or both" Hage further categorized conflict into two types: Interest conflict and Affective conflict.

Interest conflict occurs when individuals or organizations seek personal material gain or power and status, or when individuals are motivated by self-interest and the need for status and recognition. And they are inclined to pursue their interests resulting in a conflict when these are at crosspurposes with others. Affective conflict results from clashes between parties who hold different opinions, values and norms. The more dogmatic the individuals involved, the more likely it is that conflict will occur. Most disputes within institutions or organizations are a blend of interest and effective conflicts.

\section{F. Different Approaches to Conflict}

- The Radical Approach: this gained its inspiration from Hegel and Karl Marx who viewed human organization in term of the disparity in power relations, believed that conflict is a feature of unequal access to State power and resources between groups by which a conflict can never be resolved through peaceful means and measures.

- The Pluralistic Approach: This approach gained its inspiration from Frantz [12] who believes that there are different categories of people in society: youths, men, women and army are expected to play a very respective role to bring about planned change and reformation in a society. Frantz contends that "human ecology psychsocial factors" is anchored in his belief that to destroy the system of institutionalized exploitation and domination, it is lawful for the four different categories of people earlier stated to apply violence or civil disobedience.

- The Interactive Approach: According to Etekpe [13], states that 'there should be a minimal level of conflict within a group or society, to propel people to examine and re-examine their positions, and further contends that society is not a grave-yard that should be quiet at all times".

\section{G. Characteristics of Conflict}

- Nwosu [7], asserts that the characteristic of any conflict that will help us to recognize conflict situations include the following:

- When winning the conflicting battle becomes more important to the parties involved than finding a lasting solution to it.

- When the parties involved begin to conceal information from each other or to disseminate distorted information in form of misinformation or adopting negative propaganda. At this point, effective communication and mass media are urgently needed.

- When each group becomes more cohesive, and those who plan for reconciliation are punished, at this juncture strict conformity with the group position on the conflict is not only expected but demanded.

- When contact with the opposite party or group is strongly discouraged except under formalized permitted and restricted conditions.

- When the opposite party or group is negatively stereotyped, and the image or reputation of one's position or group is boosted (Effective public relations or communication is needed).

However, Ajayi [14] enlists some positive effects of conflict. He explains that if conflict is properly and promptly managed, it can achieve the following:

- Promote unity and stability when disagreements are resolved.

- Usher in innovation and better ideas in an organization or institution

- Foster interest and creativity

- Conflicting parties may be fostered to explain their views which may aid understanding and enable people 
to appreciate other people's feelings

- Offers people the opportunity to test their capabilities tolerance, forgiveness, acceptability, and peaceful coexistence.

- On the contrary, if the conflict is not resolved promptly and properly it can lead to:

- Mental in-balance: The mental health of the party (ies) may be negatively affected. In such cases, there can be skirmish, war, killing, divorce or avoidance.

- Distorted of organizational goals and this is the most important adverse effect of conflict.

- Chaos and confusion which reduces co-operation and undermines common goals, while inefficiency can be the resultant effect of conflict in any situation.

\section{H. Concept of Crisis Management}

Touitou. T.C. [3] advised that it is important to employ crisis management rather than management of the crisis, which means taking appropriate measures to prevent a crisis before it occurs. It is pro-active, preventive, and pre-planned in nature. On the other hand, "Management of crisis" literally means managing a crisis that has occurred. It involves taking sporadic, largely uncoordinated actions to quench the crisis. It is fire brigade and reactive in nature. Tools for proactive crisis management are (a) Information (b) Knowledge (c) and Communication (IKC). Hence, communication becomes more relevant when it comes to conflict and crisis management.

\section{Crisis Management Models of Managing Conflicts}

This model identifies and describes the various major forms of conflict in most societies which encapsulates the other minor forms of conflict like racial, ethnic, environmental, institutional conflict, intrapersonal, family, etc. The model exposes the various environmental factors which impinge upon these different types of conflict and which affect their occurrences, outcomes, resolution and their management. It also shows the unique place of the communication and mass media, environment and their influences in the resolution and management of conflicts. It goes further to explain that the root of all these conflicts is the individual or human beings. Therefore, this model serves as a vital part of a building block to initiate a Holistic Theory of Conflict Management, because dispute or conflict at any level needs to be managed properly in human existence.

Acceptance of conflict in human society means that individuals involved can compromise and negotiate openly to resolve the conflict. It is only when conflicts are badly handled that we have resentment, aggression or hostile attitudes that define themselves in crisis. In any case, conflicts do not always result in crisis, but it is always a strong strikers for positive change. Crisis can be defined as "any event, issue, occurrence or situation that could be described as a turning point, for good or for bad, or as "a period of heightened uncertainty that increases the need to plan, and a point in time in which external and internal pressures change objectives, operatives and operational practices of an organization, Nwosu [7]. Based on the definition, conflict or crisis has both positive and negative impacts. Both of them need proper handling through proactive conflict management approaches and principles.

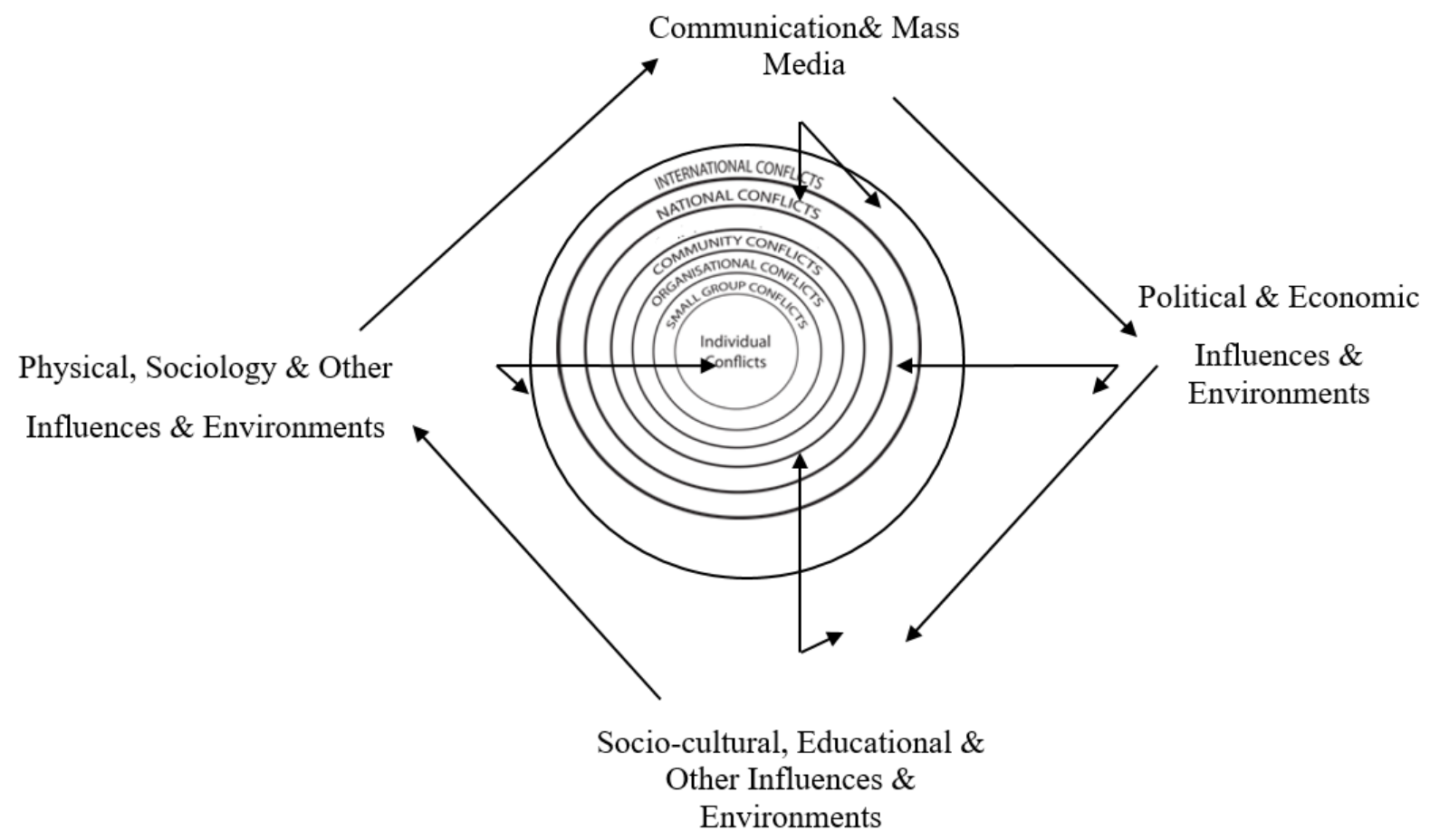

Fig. 1. Structural /Six Hubs Wheel Model of Conflict Management [7]. 


\section{J. The Structural and Process Model}

Process model views the conflict from the perspective of the structural model. It further views conflict as a progressive thing and classified conflict into four stages.

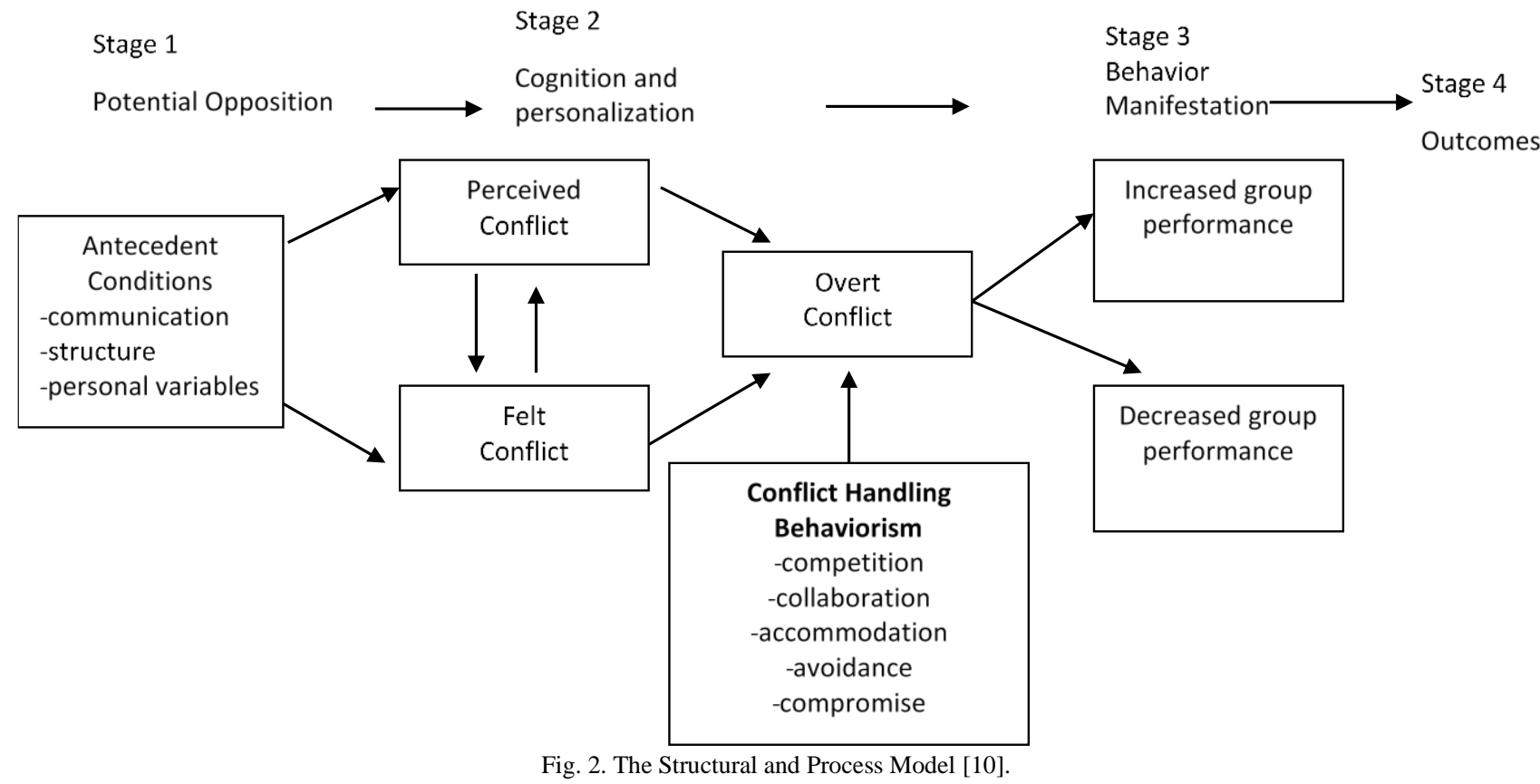

The Four Stages of Process Model are:

- Antecedent Conditions are the variables that stimulate conflict in such a situation where an individual goal is to be undermined by another. Robbins [15] identifies three sources of conflict; theyinclude communication (poor communication, noise), a structure such as size, the degree of functional specialization, jurisdictional clarity, leadership styles etc., and personal variables (individual value system) and so on and so forth.

- Cognition and Personalization of Conflict: This is said to emanate from scarce resources as when one attempts to protect his interest in a conflict situation, collective procedures and or policies and as well as role behaviours of individuals.

- Behavior Manifestation: It occurs when the conflict has become fully blown, and it is argued that expression of such conflict could manifest as subtle, inclement, aggressive, violent and uncontrolled struggle. Robbins [15], has it that when this occurs, certain forms of reaction could come in any of the following: accommodation, avoidance, competition, compromise and collaboration. Thomas, [16] and Lippit [17], further classified these reactions as withdrawal, smoothing, compromising, forcing and confrontation.

- Outcomes Are the results of conflict which could be negative or positive. The negative outcome of conflict appears more noticeable than its potential benefit. Thus, when conflict is functional, the following outcomes are assured: Sense of solidarity, the emergence of creative ideas, the formation of new policies and renewal of organization and their programs and heightened enthusiasm and purpose among the conflicting parties. On the other hand, a dysfunctional conflict precipitates: physical or psychological injury, increased hostility and misperception, hardened antagonistic positions and emotional exhaustion and so on.

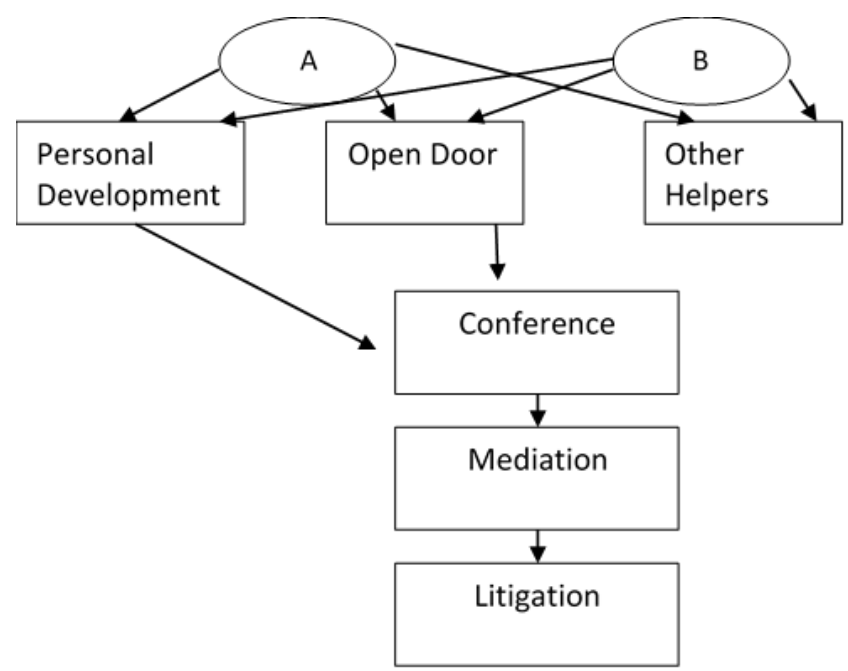

Fig. 3. Skchammer Conflict Management Model [7]

Skchammer [18] proposed a Conflict Management Program model that comprises four distinct stages:

- Open Door Stage: This aims at lowering the threshold for raising an issue, dispute or conflict to work it out promptly among the conflicting parties over parties own role, work group and immediate superior before it gets to the full-blown crisis.

- Conference Stage: This arises when the conflict could not be resolved at Open Door Stage. Organizing a conference of the conflicting parties becomes imperative because it offers a platform for communication between the parties (ASUU) versus Federal government of 
Nigeria Conflict. The objective of such a conference is not to resolve conflict and to make a joint decision as to how to process in handling the dispute. The purpose is to ascertain an optimum of procedural fairness as an important prerequisite for resolving the dispute.

- Mediation Stage: It is an internal effort on the part of the management of the conflicting organization to resolve the dispute and find an amicable settlement within its boundaries. This is more efficient and economical than litigation.

- Litigation Stage process means that representatives of the conflicting parties become involved in the conflict management process, which represents the end point.

- Conflict management system. Darling and Walker [8] synthesized these styles above as Relater, Analyzer, Director and Socializer, and he concludes that each quadrant of this model represents one of the four behavioural styles.

\section{K. Methods of Conflict Management}

Robins [19], opines that understanding and managing intentions of the parties involved in a conflict is critical as a method of conflict management. Intentions in this context refer to the decisions to act in a given conflict situation. Under these methods four distinct conflict techniques are identified:

a) Collaborating Technique: It is a win-win conflict management technique in which no one loses. It is you win; I win technique that stresses aggressive but cooperative behaviour in conflict situations. This method is necessary when the objective is to learn, to merge different perspectives or insights or to find unifying solutions.

b) Accommodating: This involves the technique of being cooperative and unassertive and used when one decides to place an individual's opponent's interest above the individual's own for one reason or the other, especially to have the conflict end at all cost. This technique is usually vital when one knows that he or she is clearly wrong or when you want a superior or better viewpoint to prevail and or when you want your subordinates to develop by learning from their mistakes in the past.

c) Compromising: It is a conflict management technique that calls for no clear winner or loser because each party to the conflict is capable and willing to giving up something in the interest of harmony, such as when goals are important but not worth the attempt.

d) Avoiding: finally, avoiding is a conflict management technique which involves being unassertive and uncooperative at the same time It is a way of ignoring or suppressing conflict with the hope that they will either disappear or remain at manageable levels without escalating. In actual sense, this technique hardly manages anything in a conflict situation. It should be used in conflict situations in which the issues are trivial or when more important issues are pressing.

\section{CONCLUSION}

An attempt has been made to look at communication, conflict and crisis management with a view to reducing or resolving conflicts and crises through effective and efficient communication. In industrial organizations as well as in larger society, conflicts and crises can be well managed before they degenerate destructive elements. It is concluded that timely use of negotiation conflict resolution and collective bargaining strategies which are in themselves communication oriented can help to promote peaceful coexistence in different organizations and in the society at large. It a true and real consensus, for communication to play its vital role in conflict resolution and crisis management. Communication should: Firstly, take a two-way asymmetric role which is about two way communication with an emphasis on persuasion; and secondly, play a two-way symmetric role where the communicators should serve as mediators between the two parties involved in a crisis. These are the true ways for communication to play its rightful-role in conflicts resolution and crises management.

\section{REFERENCES}

[1] Lester (2005): Management and Organization. Theory and Behaviour, Lagos: Molofin Nominee.

[2] Anatsui, T.C. [2015]: Public Relations Proactive Approach/; Effective Institutional Conflict Management, International Journal of Economics, Commerce and Management Vol. 111, issue 7.

[3] Touitou, T.C. [2016]: An Analysis of Speech and Communication Skills Strategies for Effective Public Relations Management for Organizational Growth in Nigeria, International Journal of International Relations, Media and Mass Communication Studies.

[4] Kunle [2005]: Communicating with Credibility and Confidence 3rd edition, (Thomson Wadsworth].

[5] Onekutu, O. \& Ojebode,A. [2007]: Trends in Health Communication research, Journal of Communication \&Language Arts, Volume 2, 2007.

[6] Moemeka, A. A. [2000]: Development Communication and the New Millennium which way for Africa? Paper presented at the 12th biennial conference of the (ACCE) 2000 Cairo, Egypt.

[7] Nwosu, I. E. [2004], Communication, Media \&Conflict Management in Nigeria, Enugu: Prime Targets Limited Publishers, p16.

[8] Darling and Walker [2001: Communication, Media \&Conflict Management in Nigeria, Enugu: Prime Targets Limited Publictionshers.p30.

[9] Kolb and Putnam (1992) Retrieved on February 7,2008 from http://en.wikipedia.org/wiki/movement.

[10] Robbin, S.P. [1989:371-81] cited in Nwosu, I. E. [2004], Communication, Media \& Conflict Management in Nigeria, Enugu: Prime Targets Limited Publishers.

[11] Hage [1980]: Cited IN Samson, N. A. (2005): The Golden Path to Conflict Prevention and Resolution, Ibadan: The Fath Centre Publication.

[12] Frantz [1986]: Cited by Etekpe (2005)"The Multiple Faces of Conflict in Organization"13,3,311-21.

[13] Etekpe [2005:29-37]: The Politics and Conflicts over Oil and Gas in Nigeria Delta Region: The Bayelsa State Experience, 1990-2006. Port Harcourt: Tower-Gate Resources.

[14] Ajayi J. F [2007]: Development is about people forwarded by Laolu Akande, wysiwyg:11421http://nigeriaworld.com/feature/speech/people.html.

[15] Robbins, S. P. [1994:170] Cited in Nwosu, I. E. [2004], Communication, Media \& Conflict Management in Nigeria, Enugu: Prime Targets Limited Publishers.

[16] Thomas, K. [1992:889-935]: Conflict Management” in Dunnette, M. Hnadbook of Industries and Organizational Psychology.Chicago:Rank Nc Nally College Publishing Company.

[17] Lippit G.L.1982): Management Conflict in todays's Organizations' Training and Development Journal 64-7.

[18] Skchammer, M. [2001]:Conflict Management in a Hospital Designing Processing Structures and Intervention Methods" Journal of Management in Medicine 15,2,1.

[19] Robins [2001]: Organiztional Behavior Concepts, Controversies, and Applications. New Delhi:Prentice-Hall India. 